\title{
CARTAN SUBALGEBRAS OF FINITE VON NEUMANN ALGEBRAS
}

\author{
ALLAN M. SINCLAIR and ROGER R. SMITH
}

\section{Introduction}

Sorin Popa [Po 1] [Po 2] has proved several results on the existence and properties of hyperfinite subfactors $R$ of a type $I I_{1}$ factor $M$ with the relative commutant $R^{\prime} \cap M$ of $R$ in $M$ equal to C1. These theorems have been used in various cohomology calculations [CES, CS, PS, CPSS], as averaging over an amenable subgroup that generates the hyperfinite subfactor is a major step in showing that the continuous and completely bounded Hochschild cohomology groups are equal. It has seemed reasonable that Popa's results could be extended from factors to general type $I_{1}$ von Neumann algebras by direct integral theory [KR, Chapter 4]. However, we do not know of such an attempt. Direct integral theory can be used directly to prove cohomology is zero and deduce results like [CPSS, Theorems 5.4 and 5.5] however these theorems on the continuous Hochschild cohomology for a von Neumann algebra with Cartan subalgebras are deduced from the theorems in this paper. This paper provides direct proofs of Popa's main two results in [Po 1] by modifying his proofs using an interpolation type result for projections in a maximal abelian selfadjoint sub-algebra (masa) of the type $I_{1}$ algebra.

This introduction contains a more detailed description of how our results extend Popa's, the basic definitions, and a brief reference to their use in the calculation of Hochschild cohomology groups in von Neumann algebras. Though averaging plays an important role in calculating the Hochschild cohomology of von Neumann algebras for all types $I, I I_{1}, I I_{\infty}, I I I$ of von Neumann algebras (see [Ri]), the results proved here are only used in the type $I I_{1}$ situation. The reason is that the type $I$ 's are already trivially hyperfinite, and the type $I I_{\infty}$ and $I I I$ von Neumann algebras may be handled by their stability under tensoring with $B(H)$. This tensor factor $B(H)$ of $M$ in the $I I_{\infty}$ and $I I I$ cases gives a suitable hyperfinite algebra over which to average. Popa [Po 1] restricts his attention to type $I I\left(I I_{1}\right.$ and $\left.I I_{\infty}\right)$ factors.

Received February 17, 1993; in final revised form February 20, 1997. 
Throughout this paper we restrict attention to type $I I_{1}$ von Neumann algebras. A maximal abelian selfadjoint subalgebra A of a von Neumann algebra $M$ is a Cartan subalgebra if the von Neumann algebra generated by the unitary normaliser $\left\{u \in \mathscr{U}(M): u A u^{*}=A\right\}$ of $\mathrm{A}$ in the unitary group $\mathscr{U}(M)$ of $M$ is equal to $M$ [Po 1]. A von Neumann subalgebra $N$ of a von Neumann algebra $M$ with centre $Z$ is said to have trivial relative commutant if $N^{\prime} \cap M=Z$.

The two results of [Po 1] that we generalise (Theorems 8 and 9) from type $I I_{1}$ factors to general type $I I_{1}$ von Neumann algebras with separable predual are the following.

Each type $I I_{1}$ factor $M$ with separable predual contains a hyperfinite subfactor $N$ such that $N$ has trivial relative commutant in $M$.

For each Cartan subalgebra $A$ in a type $I I_{1}$ factor $M$ with separable predual there is a hyperfinite subfactor $N$ with trivial relative commutant such that $A$ is a Cartan subalgebra of $N$.

Note that the counter example in [Po 1, Section 4] shows that the hypothesis "with separable predual" is necessary.

The technical lemmas on the comparison of projections in a masa are stated and proved in Section 2. The proofs of these lemmas are modifications of standard results on the comparison of projections in von Neumann algebras. Observe that though all the projections are in an abelian subalgebra, the techniques are highly non-commutative as the equivalence is calculated in the whole algebra. Throughout $M$ is taken to be a type $I I_{1}$ von Neumann algebra with centre $Z$ and centre valued trace $T$ [Ta 2]. The separable predual condition implies that there is a faithful normal state $\rho$ on $Z$, and $\tau=\rho o T$ is a faithful normal tracial state on $M$. Let $\|x\|_{2}=\tau\left(x^{*} x\right)^{1 / 2}$ for all $x \in M$, and let $L^{2}(M)$ be the completion of $M$ in this norm. Since $\tau$ is a normal tracial state, a result of Takesaki [Ta 1] [St] implies that for each unital von Neumann subalgebra $N$ of $M$ there is a $\tau$ preserving conditional expectation $E_{N}$ from $M$ onto $N$.

Section 3 contains the statement and proof of Theorem 8. This result shows that for each type $I I_{1}$ von Neumann algebra there is a hyperfinite subalgebra $N$ with trivial relative commutant. This conclusion is the input required into the averaging arguments used in the applications of Grothendiecks's inequality to cohomology computations (see [ES], [PS], [CPSS]).

Theorem 9, and a lemma on the construction of matrix units associated with a Cartan subalgebra, are stated and proved in Section 4. The result enables one to avoid the use of direct integral theory in the proof that $H_{c}^{3}(M, M)=0$ for a type $I I_{1}$ von Neumann algebra $M$ with Cartan subalgebra [CPSS, Theorem 6.4]. One just needs to observe that the conclusion 
of Theorem 9 can be used in place of Popa's result [Po 1] in the proof of [CPSS, Theorem 5.5].

The proofs of Theorems 8 and 9 are rather similar with one important difference which occurs in Popa's arguments [Po 1]. As the $k_{n} \times k_{n}$ matrix units are constructed at the $n^{\text {th }}$ stage, they perturb the fine structure of the initial masa. In Theorem 8 this means the masa is changing from the $n^{\text {th }}$ to the $(n+1)^{t h}$ stage of the construction. There is little control over the final masa. This difficulty is avoided in the Cartan algebra situation in Theorem 9 by ensuring that the partial isometries chosen leave the masa invariant (Lemma 10).

The authors were partially supported by a NATO collaborative research grant and the second author was partially supported by a National Science Foundation grant. The authors wish to thank the organisers of the Symposium on Invariants in Operator Algebras at the Royal Danish Academy of Science and Letters in August 1992; participation in this symposium enabled the authors to discuss this research. The first author would like to thank Erik Christensen for many stimulating conversations about type $I I_{1}$ von Neumann algebras. The authors wish to thank the referee for helping us correct certain errors and ambiguities.

\section{Finite dimensional subalgebras of masas}

This section contains the basic lemmas on constructing nice finite dimensional subalgebras of masas in type $I I_{1}$ von Neumann algebras. Throughout this section we shall assume that $M$ is a type $I I_{1}$ von Neumann algebra with separable predual and with centre $Z$, and that $\mathrm{A}$ is a masa in $M$. The centre valued tracial conditional expectation will be denoted by $T$ (see [Ta 2], [Di], [SZ]). Since $M$ has separable predual, so does $Z$, and there is a faithful normal state $\rho$ on $Z$. Let $\tau=\rho \circ T$ be the resulting faithful tracial state on $M$, and let $\|x\|_{2}=\left(\tau\left(x^{*} x\right)\right)^{1 / 2}$ for all $x \in M$. If $C$ is a von Neumann subalgebra of $M$, let $E_{C}$ denote the $\tau$-preserving conditional expectation from $M$ onto $C$ [Ta2 ] [ St ], and let $P(C)$ denote the set of projections in $C$. Recall that the $\|\cdot\|_{2}$ topology on the closed unit sphere $M_{1}=\{x \in M:\|x\| \leq 1\}$ in $M$ is equivalent to the strong and ultrastrong topology on $M_{1}$ [Ta 1] [Di].

Throughout equivalence of projections $e \sim f$ is equivalence within $M$, i.e. there is a $v$ (a partial isometry) in $M$ such that $v^{*} v=e$ and $v v^{*}=f$. Recall that the order $\preccurlyeq$ on projections is defined by $e \preccurlyeq f$ if and only if there is a projection $g$ with $e \sim g \leq f$.

Lemmas 1 to 4 are modifications of the standard comparison theory lemmas for projections in von Neumann algebras but taking into account that the projections lie in the masa A. Lemma 3 provides the crucial interpolation 
step that enables one to choose a projection in the masa between two other projections in a suitable way to continue the inductive construction of the increasing sequence of finite dimensional algebras.

Lemma 1 (SZ, 4.5). If $h(\neq 0) \in P(A)$, then there are $f_{1}, f_{2}$ in $P(A)$ such that $f_{1} \leq h, f_{2} \leq h, f_{1} f_{2}=0$ and $f_{1} \sim f_{2}$.

Proof. Choose a $g \in P(A)$ such that $g \leq h, g \neq 0, g \neq h$ and $g \notin Z h$. This choice of $g$ is possible, because $h A$ is a masa in $h M h$, which is a type $I I_{1}$ von Neumann algebra so that

$$
h A \supsetneqq Z(h M h)=\text { the centre of } h M h \supseteq h Z \text {. }
$$

By the Comparison Theorem for projections in von Neumann algebras [SZ, Theorem 4.6] applied to $M$, there is a $p \in P(Z)$ such that

$$
g p \preccurlyeq(h-g) p \text { and } g(1-p) \succcurlyeq(h-g)(1-p)
$$

Now either $g p \neq 0$ or $(h-g)(1-p) \neq 0$; because if $g p=0$ and $h-g-h p+g p=0$, then $g=h(1-p) \in Z(h M h)$ contrary to the assumption $g$ is not in $Z(h M h)$.

Let $f_{1}$ be either $g p$ or $(h-g)(1-p)$, whichever is non zero. Let

$f_{2} \sim g p$ with $f_{2} \leq(h-g) p$ if $f_{1}=g p$, or

$f_{2} \sim(h-g)(1-p)$ with $f_{2} \leq g(1-p)$ if $f_{1}=(h-g)(1-p)$.

In the first case $f_{1} f_{2} \leq g p \cdot(h-g) p=0$ and in the second case $f_{1} f_{2} \leq(h-g)(1-p) g(1-p)=0$. Further $f_{1} \leq h$ and $f_{2} \leq h$. (Recall in these last calculations that all our projections are in A and $h g=g$.)

Lemma 2 (SZ, 4.11). If $e \in P(A)$, then there are $e_{1}, e_{2} \in P(A)$ such that $e=e_{1}+e_{2}, e_{1} \sim e_{2}, e_{1} e_{2}=0$.

Proof. Let $\mathscr{F}=\left\{\left(e_{1 i}, e_{2 i}\right): i \in I\right\}$ be a maximal family of pairs of projections in $A$ such that $e_{1 i} \leq e, e_{2 i} \leq e$ and $e_{1 i} \sim e_{2 i}$ for all $i \in I$, and $e_{1 i} \cdot e_{2 j}=0$ for all $i, j \in I$; where $\mathrm{I}$ is a suitable index set. Let $e_{1}=\vee e_{1 i}$ and $e_{2}=\vee e_{2 i}$. Then $e_{1} \sim e_{2}$ and $e_{1} e_{2}=0$ by standard von Neumann algebra projection theorems [SZ, Theorem 4.2]. If $h=e-e_{1}-e_{2} \neq 0$, then by Lemma 1 there exists $f_{1}, f_{2} \in P(A)$ with $0 \neq f_{1} \leq h, f_{2} \leq h, f_{1} \sim f_{2}$ and $f_{1} \cdot f_{2}=0$. Further $f_{1} \cdot e_{2 i}$ and $f_{2} \cdot e_{1 i}=0$ for all $i \in I$. Thus the pair $\left(f_{1}, f_{2}\right)$ may be adjoined to the family $\mathscr{F}$ contradicting the maximality of $\mathscr{F}$. Hence $e=e_{1}+e_{2}$ as required.

The interpolation in $A$ used is that of the following lemma. For a factor the following lemma is just the fact that given two projections $e \leq f$ in the masa $A$ and a real number $\lambda$ with $\operatorname{tr}(e) \leq \lambda \leq \operatorname{tr}(f)$ there is a projection $g$ in A with $e \leq g \leq f$ and $\operatorname{tr}(g)=\lambda$. If the hypothesis "in $A$ " is dropped, then this fact is well known dating back to Murray and von Neumann. 
Lemma 3 (SZ, 7.17). Let $e \leq f \in P(A)$. If $z \in Z$ with $T e \leq z \leq T f$, then there is a $g \in P(A)$ with $e \leq g \leq f$ and $T g=z$.

Proof. Let $\mathscr{F}$ be the family of all $h \in P(A)$ such that $e \leq h \leq f$ and $T h \leq z$, and let $\mathscr{F}$ have the order induced on it from the projection lattice $P(A)$. Let $\mathscr{F}_{o}$ be a maximal totally ordered subfamily of $\mathscr{F}$ and let $g=\vee\left\{h: h \in \mathscr{F}_{o}\right\}$. By the total order of $\mathscr{F}_{o}$ and the positivity and the ultraweak continuity of $T, e \leq g \leq f$ and $T g \leq z$.

Thus $g \varepsilon \mathscr{F}_{o}$. Suppose that $z-T g \neq 0$. By standard von Neumann algebra techniques (the comparison theorem et al) there is an $\varepsilon>0$ and a non-zero $p \in P(Z)$ such that $(z-T(g)) p \geq \varepsilon p$ (if $\varepsilon$ and $p$ do not exist, then $z-T g=0)$. Now $(f-g) p \varepsilon P(A),(f-g) p . \quad g=0$ and $T((f-g) p)=$ $(T f-T g) p \geq(z-T g) p \geq \varepsilon p$, since $p \in Z$. Hence $(f-g) p \neq 0$. Let $n \in \mathrm{N}$ with $2^{-n} \leq \varepsilon$.

Repeated application of Lemma 2 to the projection $(f-g) p$ in $A$ shows that there are mutually orthogonal equivalent projections $e_{1}, e_{2}, \ldots, e_{2^{n}}$ in $A$ such that $(f-g) p=\Sigma e_{j}$. The equivalence implies that $T\left(e_{j}\right)=T\left(e_{k}\right)$ for all $j, k$ (by [SZ, Theorem 7.11], [Ta, Theorem 2.6]). Hence

$$
T\left(e_{1}\right)=2^{-n} T((f-g) p) \leq 2^{-n} p \leq \varepsilon p .
$$

since $T(f-g) \leq 1$. Note that $e_{1} g=0$ so $g+e_{1} \in A$. Further

$$
\begin{aligned}
e \leq g+e_{1} & \leq g+(f-g) p=g(1-p)+f p \\
& \leq f(1-p)+f p=f,
\end{aligned}
$$

and

$$
\begin{aligned}
T\left(g+e_{1}\right) & \leq T(g)+\varepsilon p \leq T(g)+(z-T(g)) p \\
& \leq z p+T(g)(1-p) \leq z .
\end{aligned}
$$

Thus $g+e_{1} \in \mathscr{F}_{o}$ and this contradicts the maximality of $g$ in $\mathscr{F}_{o}$, so $z=T g$ as required.

Lemma 4. Let $f \in P(A), \quad p \in P(Z)$ and $0<r<m$ be integers. If $T f=r m^{-1} p$, then there are orthogonal equivalent projections $e_{1}, \ldots, e_{r}$ in $A$ with $f=\Sigma_{1}^{r} e_{j}\left(\right.$ and $\left.T e_{j}=m^{-1} p\right)$.

Proof. The projections $e_{1}, \ldots, e_{r}$ are constructed inductively. By Lemma 3 there is $e_{1} \leq f$ with $T e_{1}=m^{-1} p$. If orthogonal projections $e_{1}, \ldots, e_{t}(t<r)$ have been constructed with $T e_{j}=m^{-1} p$, then $T\left(f-\Sigma_{1}^{t} e_{j}\right)=(r-t) m^{-1} p$ so there is a projection $e_{t+1}$ with $T e_{t+1}=m^{-1} p$ and $e_{t+1} \leq f-\Sigma_{1}^{t} e_{j}$. This gives orthogonal projections $e_{1}, \ldots, e_{r}$ with $f=\Sigma_{1}^{r} e_{j}$ and $T e_{j}=m^{-1} p$; the equivalence of $e_{j}$ follows from them having equal central trace [SZ],[Ta1].

Note that although the next lemma involves approximation in a masa $A$ in $M$ it is not a commutative result, because the equivalence is that in $M$. 
Lemma 5, Let $B$ be a finite dimensional subalgebra of $A$ such that there is an integer $k$ with the property that if $q$ is a minimal projection in $B \cap Z$, then there are exactly $k$ minimal projections in $q B$ and they are all equivalent (in $M$ ). Let $\varepsilon>0$ and let $g_{1}, \ldots, g_{n}$ be projections in $A$. Then there is a finite dimensional subalgebra $A_{o}$ of $A$ that contains $B$ and has the property that there is a positive integer $m$ so that

1. $k$ divides $m$,

2. if $p$ is a minimal projection in $A_{o} \cap Z$, then there are exactly $m$ minimal projections in $A_{o}$ and they are all equivalent (in $M$ ), and

3. $\left\|g_{j}-E_{A_{o}} g_{j}\right\|_{2} \leq \varepsilon$ for $1 \leq j \leq n$.

Note that standard lemmas on approximating projections [Co] and [Ch] imply that the $y_{j}$ could be chosen to be projections in $A_{o}$, but we do not require this subsequently so do not follow this up.

The condition on the minimal projections in the centre giving rise to equivalent minimal projections below may be written symbolically for $B$ : if $q$ is a minimal projection in $Z \cap B$, then there are equivalent minimal projections $e_{1}, \ldots, e_{k}$, in qB with $e_{1} \sim e_{j}$ for $1 \leq j \leq k$. In the proof below no effort is made to control the values of $k$. However, a little more care shows that if $k$ is a power of 2 , then $m$ can be chosen to be a power of 2 .

Proof. The proof splits into a particular preliminary case with $B \cap Z=\mathrm{C}$ 1, which is the main part of the proof, and the general case, which is just a finite sum of the particular ones. For the first part of the proof (most of it) assume that $B \cap Z=C 1$ and that $B$ is spanned by its equivalent minimal projections $u_{j}(1 \leq j \leq k)$; they are equivalent by hypothesis. Thus the centre valued trace $T$ has $T u_{j}=k^{-1} .1$, since $T u_{j}$ is a multiple of 1 .

Let $C_{0}$ be the finite dimensional *-subalgebra of $A$ generated by $g_{1}, \ldots, g_{n}$ and $B$. Let $m_{0}$ be a positive integer such that $m_{0} \geq 2^{n+1} \varepsilon^{-2}$ and let $m=m_{0} k$.

If $F$ is a finite set of projections, let $\mathrm{N} \operatorname{span}(F)$ denote the additive semigroup generated by $F \cup\{0\}$, and if $r$ is a positive integer, let $r^{-1} \mathrm{~N} \operatorname{span}(F)$ denote this semigroup times $r^{-1}$. The abelian von Neumann algebras with separable preduals are isomorphic to $L^{\infty}[0,1], \ell^{\infty}(\mathrm{N}), \ell^{\infty}(r)$ for $r \in \mathrm{N}$, or to the direct sum of the first with one of the other two [Ta1, p.112]. Hence the real linear span of the set of projections in $Z$ is dense in the self-adjoint part of $Z$ in the norm topology, and the set $\left\{x=x^{*} \in Z: x \geq 0\right\}$ is covered by the open sets

$$
\left\{x=x^{*} \in Z: x \geq 0,\|x-y\|<m^{-1} \text { for some } y \in m^{-1} \mathrm{~N} \operatorname{span}(F)\right\}
$$

as $F$ runs over all finite sets of pairwise orthogonal projections in $Z$ that add to 1 . The set $\left\{w \in T C_{0}: 0 \leq w \leq 1\right\}$ is compact in the norm topology, since $C_{0}$ is finite dimensional. Hence there is a finite set $F_{0}$ of projections in $Z$ such 
that for each $w \in T C_{0}$ with $0 \leq w \leq 1$ there is a $v$ in $m^{-1} \mathrm{~N} \operatorname{span}\left(F_{0}\right)$ with $\|w-v\|<m^{-1}$. If the projections in $F_{0}$ are multiplied by the minimal projections in $T C_{0}$, we obtain a finite set $F$ of orthogonal projections in $Z$ adding to 1 with the properties that

(1) if $w \in T C_{0}$ with $0 \leq w \leq 1$, then there is a $v$ in $m^{-1} \mathrm{~N} \operatorname{span}(F)$ with $\|v-w\|<m^{-1}$, and

(2) each projection in $T C_{0}$ is a sum of projections in $F$.

Let $C$ be the finite dimensional subalgebra of $A$ generated by $C_{0}$ and $F$. Observe that each central projection in $T C_{0}$ is a sum of orthogonal projections in $F$, so $F$ is the set of minimal projections in $Z \cap C$. Fix a projection $p$ in $F$ and a minimal projection $u\left(=u_{j}\right)$ in $B$. Since $p u$ is a projection in the commutative finite dimensional algebra $p C$, there are minimal projections $f_{1}, \ldots, f_{\ell}$ in $p C$ so that $p u=\sum_{1}^{\ell} f_{j}$. The dependence of $\ell$ and $f_{j}$ on $u$ and $p$ is omitted here to simplify notation. Note that $p u$ is not zero, since $T(p u)=p T(u)=k^{-1} p$. Note that the number of minimal projections in $C$ that add to each minimal central projection in $C$ (i.e. $p$ in $F$ ) is no more than the corresonding maximal number adding to a central projection in $C_{0}$, because $C$ is generated by $C_{0}$ and $F \subset Z$. This is because multiplying by a central projection does not increase this number. The projections $g_{j}$ may not be in $Z$ so each pair $\left(g_{j}, 1-g_{j}\right)$ can split a minimal projection in two. Hence the number of minimal projections in $C$ below $p u$ is no more than $2^{n}$, because $u$ is minimal in $B$ and $p$ is minimal central in $C$. Thus $\ell \leq 2^{n}$.

Now $T(p u)=p T(u)=k^{-1} p$. Also $p u f_{j}=p f_{j}=f_{j}$ implies that $0 \leq f_{j} \leq$ $p u \leq 1$ so that

$$
0 \leq T f_{j}=p T f_{j} \leq T(p u)=k^{-1} p
$$

for $j=1, \ldots, \ell$. Thus $T f_{j}$ can be approximated in norm by a non-negative rational multiple of $p$ with denominator $m$ by (1), the choice of $F$ and $F_{0}$, and the minimality of $p$ in $C \cap Z$. Hence there is an integer $r_{j}$ with $0 \leq r_{j} \leq m$ so that $\left\|T f_{j}-m^{-1} r_{j} p\right\|<m^{-1}$. As $T f_{j} \geq 0$, this is equivalent to

$$
0 \leq m^{-1} \max \left\{0,\left(r_{j}-1\right)\right\} p \leq T f_{j} \leq m^{-1}\left(r_{j}+1\right) p .
$$

If $T f_{j}=m^{-1} r_{j} p$, choose $e_{j}=f_{j}$. If $T f_{j} \neq m^{-1} r_{j} p$, then there is a projection $e_{j}$ in $A$ with $e_{j} \leq f_{j}$ and $\mathrm{Te}_{j}=m^{-1} \max \left\{0,\left(r_{j}-1\right)\right\} p$ by Lemma 3. Thus $0 \leq T\left(f_{j}-e_{j}\right) \leq 2 m^{-1} p$ for each $j$. Let $e_{0}=p u-\sum_{1}^{\ell} e_{j}$. Note that $e_{0}, \ldots, e_{\ell}$ are orthogonal projections, because $f_{1}, \ldots, f_{\ell}$ are orthogonal projections with $e_{j} \leq f_{j}(1 \leq j \leq \ell)$, and that any of the $e_{j}$ could be zero. The central trace of $e_{0}$ satisfies 


$$
T e_{0}=k^{-1} p-\sum_{1}^{\ell} T\left(e_{j}\right)=r_{0} m^{-1} p
$$

for some non-negative integer $r_{0}$, since $T(p u)=k^{-1} p=m_{0} m^{-1} p$. By Lemma 4 each projection $e_{j}(0 \leq j \leq \ell)$ may be subdivided into orthogonal equivalent projections $e_{j i}$ with $T\left(e_{j i}\right)=m^{-1} p$ for all $j$ and $i$. The projections $e_{j i}$ are orthogonal equivalent and add up to $p u$. There are $m_{0}$ of these projections $e_{j i}$ for each $p u$ since $T(p u)=k^{-1} p=m_{0} m^{-1} p$.

Note that $p=p u_{1}+\ldots+p u_{k}$, so that there are $m=k m_{0}$ orthogonal equivalent projections $e_{j i}^{p, 1}, \ldots, e_{j i}^{p, k}$ that add to $p$; denote these projections by $h_{s}^{p}$ for $1 \leq s \leq m$.

Let $A_{0}$ be the linear span of $\left\{h_{s}^{p}: p \in F, 1 \leq s \leq m\right\}$ so that $A_{0}$ is a commutative finite dimensional $*$-subalgebra of $A$. For each $p u$ above, each $e_{j}$ is in $A_{0}$ by construction of $h_{s}^{p}$. Hence for each $u_{j}$ in $B(1 \leq j \leq k)$ and each $p$ in $F, p u_{j}$ is in $A_{0}$ as it is a sum of $e_{i}$ by construction of $e_{0}$. Hence $u$ is in $A_{0}$ as $u=\sum\{p u: p \in F\}$. When constructed $F$ was observed to be the set of minimal projections in $C \cap Z$ and each $p$ in $F$ was shown to be the sum of $m$ orthogonal equivalent projections $h_{s}^{p}(1 \leq s \leq m)$. The equivalence of $h_{s}^{p}$ and $h_{t}^{p}$ for $1 \leq s, t \leq m$ imply that a sum $\sum h_{j}^{p}$ for fixed $p$ over some $j$ is in the centre $Z$ if and only if it is over all $1 \leq j \leq m$. This implies that $F$ is the set of minimal projections in $A_{0} \cap Z$ and that each $p$ in $F$ is the sum of $m$ equivalent (orthogonal) minimal projections in $p A_{0}$. This proves properties 1 and 2 .

We now turn to proving property 3 . Let $g$ be a projection in $C$. Since $g$ is a sum of minimal projections in $C$, there is a subset $W$ of

$$
\left\{\left(p, f_{j}^{p}\right): p \in F, f_{j}^{p} \text { is a minimal projection in } p C\right\} \text {; }
$$

such that

$$
g=\sum\left\{f_{j}^{p}:\left(p, f_{j}^{p}\right) \in W\right\} .
$$

Let $h=\sum\left\{e_{j}^{p}:\left(p, f_{j}^{p}\right) \in W\right\}$ where $e_{j}^{p}$ are the projections constructed corresponding to the projections $f_{j}^{p}$. Note that the $e_{j}^{p}$ are not minimal projections in $A_{0}$ but are each sums of equivalent minimal projections in $A_{0}$. By construction $e_{j}^{p} \leq f_{j}^{p}$ for all $j$ and $p$, so $h$ is a projection in $\mathrm{A}_{0}$ with $h \leq g$. Hence

$$
\|g-h\|_{2}^{2}=\tau(g-h)=\sum \tau\left(p\left(f_{j}^{p}-e_{j}^{p}\right)\right)=\sum \rho\left(p \cdot T\left(f_{j}^{p}-e_{j}^{p}\right)\right)
$$

by the properties of $T$ and $\tau=\rho T$ with the above sums over $\left(p, f_{j}^{p}\right) \in W$. For each $j, 0 \leq p T\left(f_{j}^{p}-e_{j}^{p}\right) \leq 2 m^{-1} p$ and for each $p$ there are at most $2^{n} k$ such elements because this is the maximal number of minimal projections in $C$ below a minimal $p$ in $C \cap Z$. Hence using the minimality properties of the fact that $E_{A_{0}}$ is the orthogonal projection onto $A$ in the Hilbert space $\left(M,\|\cdot\|_{2}\right)$ 


$$
\left\|g-E_{A_{0}} g\right\|_{2}^{2} \leq\|g-h\|_{2}^{2} \leq \sum \rho(p) \cdot 2 \cdot m^{-1} \cdot 2^{n} k
$$

where the sum is over $p$ in $\mathrm{F}$.

Thus

$$
\left\|g-E_{A_{0}} g\right\|_{2}^{2} \leq m^{-1} \cdot 2^{n+1} k=m_{0}^{-1} \cdot 2^{n+1} \leq \varepsilon^{2}
$$

by the choice of $m_{0}$ and $m=m_{0} k$.

This proves the case where $B \cap Z=\mathrm{C} 1$.

In general $B \cap Z$ is a finite dimensional commutative $C^{*}$-algebra with minimal projections $q_{1}, \ldots, q_{N}$. Cutting the projections $g_{1}, \ldots, g_{n}$ by a central projection $q_{t}$ gives $n$ projections $g_{1} q_{t}, \ldots, g_{n} q_{t}$ in the algebra $A q_{t}$ with $B q_{t} \cap Z q_{t}=\mathrm{C} q_{t}$ and $q_{t}$ the identity of the von Neumann algebra $q_{t} M$. Since $\rho(1)=1=\sum \rho\left(q_{t}\right)$, the approximations obtained in the various factors add to the whole algebra provided $\rho$ is replaced on $M q_{t}$ by $\rho\left(q_{t}\right)^{-1} \rho(\cdot)$. Properties 1 and 2 follows directly as they concern elements below minimal projections in $B \cap Z$. Property 3 follows because of orthogonality of $\|\cdot\|_{2}$-norm on the direct sum $M=\bigoplus M q_{t}$ and the choice of state $\rho\left(q_{t}\right)^{-1} \rho(\cdot)$ on $M q_{t}$. This completes the proof.

The following lemma is just the inductive version of Lemma 5; however, in the form given below it fits in better with the Popa's characterization of masa's in von Neumann algebras [Po1, Lemma 1.2].

Lemma 6. Let $A_{o}$ be a finite dimensional subalgebra of $A$ so that there is a positive integer $k_{o}$ with the property that if $p$ is a minimal projection in $A_{o} \cap Z$, then there are exactly $k_{o}$ minimal projections in $P A_{o}$ and they are all equivalent (in $M$ ). Then there is a sequence $A_{n}$ of finite dimensional subalgebras of $A$ for $n \in \mathrm{N}$ such that

1. $A_{n-1} \subseteq A_{n}$ for $n \geq 1$,

2. $k_{n-1}$ divides $k_{n}$ for $n \geq 1$,

3. if $q$ is a minimal projection in $A_{n} \cap Z$, then there are exactly $k_{n}$ minimal projections in $q A_{n}$ and they are all equivalent, and

4. the weak closure of the union of the $A_{n}$ is $A$, i.e. $\left(\cup A_{n}\right)^{-w}=A$.

Proof. The von Neumann algebra $A$ has a separable predual, since $M$ has a separable predual, so there is a sequence $\left\{g_{n}: n \in \mathrm{N}\right\}$ of projections in $A$ such that the linear span of $\left\{g_{n}: n \in \mathrm{N}\right\}$ is weakly dense in $A$.

By induction we choose an increasing sequence $A_{n}$ of finite dimensional subalgebras of $A$ satisfying (1), (2) and (3) of our conclusions above, and such that

5. $\left\|g_{j}-E_{A_{n}} g_{j}\right\|_{2} \leq 2^{-n}$ 
for $1 \leq j \leq n$. Observe that Lemma 5 gives the step from $n-1$ to $n$ in the induction. Property (4) follows from inequality (5).

The next lemma is used in the proofs of Theorems 8 and 9 to show that the hyperfinite algebras constructed there have trivial relative commutants.

Lemma 7. Let $\mathrm{C} 1=N_{o} \subseteq N_{2} \subseteq \ldots$ be an increasing sequence of finite dimensional *-subalgebras of $M$ such that the centre of $N_{n}$ is equal to $N_{n} \cap Z$ for all $n$. Let $N$ be the weak closure of the union of $N_{n}$, i.e. $N=\left(\cup N_{n}\right)^{-w}$. If $N$ contains $Z$, then $N \cap N^{\prime}=Z$.

Proof. Let $x=x^{*} \in N \cap N^{\prime}$ with $\|x\| \leq 1$. The strong topology and the $\|.\|_{2}$-topology coincide on the closed unit ball $M_{1}$ of $M$. Hence, by the Kaplansky density theorem [Ta 1], there is a $n \in \mathrm{N}$ and $y \in N_{n}$ such that $\|y\| \leq 1$ and $\|x-y\|_{2}<\varepsilon$. Since $x \in N^{\prime} \subseteq N_{n}^{\prime}$, $\|y u-u y\|_{2}<2 \varepsilon$ for all $u \in N_{n}$ with $\|u\| \leq 1$. Thus $\left\|y-u y u^{*}\right\|_{2} \leq 2 \varepsilon$ for all $u$ in the unitary group $\mathscr{U}\left(N_{n}\right)$ of $N_{n}$. Let $\mu$ denote normalised Haar measure on the (compact) unitary group $\mathscr{U}\left(N_{n}\right)$. Let $z=\int\left(y-u y u^{*}\right) d \mu(u)$, with integration over the whole of the unitary group $\mathscr{U}\left(N_{n}\right)$. Then $\|z\|_{2} \leq 2 \varepsilon$ and $w(y-z) w^{*}=\int w u y u^{*} w^{*} d \mu(u)=$ $\int v y v^{*} d \mu(v)=y-z$ for all $w \in \mathscr{U}\left(N_{n}\right)$. Hence $y-z$ is in the centre of $N_{n}$, which is equal to $Z \cap N_{n}$. Further $\|x-(y z)\|_{2} \leq 3 \varepsilon$; this shows that $x$ is in $\|\cdot\|_{2}$ closure of $Z$, so is in $Z$.

\section{Injective subalgebras with trivial relative commutant}

THEOREM 8. Let $M$ be a type $I_{1}$ von Neumann algebra with separable predual and centre $Z$. Then there is a masa $A$ in $M$ and an injective von Neumann subalgebra $N$ of $M$ containing $A$ such that $A$ is a Cartan subalgebra of $N$ and $N$ has trivial relative commutant in $M$.

Proof. Let $\left\{x_{j}: j \in \mathrm{N} \cup\{0\}\right\}$ be a subsequence of the closed unit ball $M_{1}$ of $M$ that is dense in this unit ball in the $\|\cdot\|_{2}$ norm; such a sequence exists by the separability of the predual of $M$ and the equivalence of the $\|\cdot\|_{2}$-topology and the strong topology on $M_{1}$. We shall assume $x_{o}=1$. By induction on $n$ we shall construct sequences

$$
A_{o}=\mathrm{C} 1 \subseteq A_{1} \subseteq A_{2} \subseteq \ldots
$$

of finite dimensional abelian subalgebras of $M$,

$$
N_{o}=\mathrm{C} 1 \subseteq N_{1} \subseteq N_{2} \subseteq \ldots
$$

of finite dimensional *-subalgebras of $N$, and

$$
k_{o} \leq k_{1} \leq k_{2} \leq \ldots
$$

of positive integers such that

1. $A_{n} \subseteq N_{n}$,

2. the centre of $N_{n}$ is $N_{n} \cap Z$, which is contained in $A$,

3. $k_{n-1}$ divides $k_{n}$, 
4. for each minimal projection $q$ in $Z \cap A_{n}$, there are exactly $k_{n}$ minimal projections in $q A_{n}$ and they are all equivalent,

5. for each minimal projection $q$ in $Z \cap A_{n}$, the algebra $q N_{n}$ is isomorphic to $\mathrm{M}_{k_{n}}(\mathrm{C})$ and has matrix units $e_{i j}^{q}$ so that $e_{i i}^{q}\left(1 \leq i \leq k_{n}\right)$ are the minimal projections of $q A_{n}$,

6. for each $1 \leq j \leq n-1$ and each minimal projection $p$ in $A_{j} \cap Z$, the natural unitary in $N_{j}$ that interchanges $e_{11}^{p}$ and $e_{i i}^{p}$ normalises $A_{n}$, and

7. $\left\|\left(E_{A_{n}^{\prime} \cap M}-E_{A_{n}}\right)\left(x_{i}\right)\right\|_{2} \leq 2^{-n}$ for $1 \leq i \leq n$.

The induction starts with $A_{o}=N_{o}=C 1$ and $k_{o}=1$. Suppose that $A_{n}, N_{n}$ and $k_{n}$ have been constructed as above. Let $B$ be a maximal abelian subalgebra of $M$ containing $A_{n}$. By Lemma 2.6 with $A_{o}$ there equal to $A_{n}$ choose an increasing sequence $B_{\ell}$ of finite dimensional subalgebras of $B$ containing $A_{n}$ with the properties of that lemma. As the algebra $B$ is a masa in $M$ and $B=(\cup B)^{-w},[$ Po 1, Lemma 1.2] implies that

$$
\left\|E_{B_{\ell}^{\prime} \cap M}(x)-E_{B_{\ell}}(x)\right\|_{2} \rightarrow 0
$$

as $\ell \rightarrow \infty$ for all $x \in M$. For each minimal projection $p$ in $Z \cap A_{n}$, let $e_{i j}^{p}\left(1 \leq i, j \leq k_{n}\right)$ be the matrix units in the algebra $p N_{n}$. Let $\delta_{n}$ be the number of minimal projections in $Z \cap A_{n}$. Choose $\ell$ so large that

8. $\left\|\left(E_{B_{\ell}^{\prime} \cap M}-E_{B_{\ell}}\right)\left(e_{i j}^{p} x_{i} e_{j i}^{p}\right)\right\|_{2}<2^{-(n+1)} \delta_{n}^{-\frac{1}{2}} k_{n}^{-\frac{1}{2}}$

for $1 \leq i \leq n+1,1 \leq j \leq k_{n}$ and all minimal projections $p$ in $Z \cap A_{n}$. Let $Q_{n+1}$ be the set of all minimal projections in this $B_{\ell} \cap Z$. Now for each $q \in Q_{n+1}$ the projection $q e_{11}^{p}$ is a sum of $m_{n}=k_{n+1} / k_{n}$ minimal projections in $q B_{\ell}$ by Lemma 6 , where $k_{n+1}$ is the integer corresponding to $B_{\ell}$ of that lemma. Number these $m_{n}$ minimal projections in $q B_{\ell}$ as $e_{i i}^{q}$ for $I \leq i \leq m_{n}$. For each $q$, the projections $e_{i i}^{q}\left(1 \leq i \leq m_{n}\right)$ are equivalent in $M$ so form the diagonal of a set of matrix units $e_{i j}^{q}\left(1 \leq i, j \leq m_{n}\right)$ in $M$.

Let $A_{n+1}$ be the linear subspace of $M$ spanned by the set $\left\{e_{j 1}^{p} e_{t t}^{q} e_{1 j}^{p}: 1 \leq t \leq m_{n}, \quad 1 \leq j \leq k_{n}, q \in Q_{n+1}, \quad p\right.$ the minimal projection in $Z \cap A_{n}$ with $\left.q=q p\right\}$.

Let $N_{n+1}$ be the linear subspace of $M$ spanned by the set

$\left\{e_{i 1}^{p} e_{s t}^{q} e_{1 j}^{p}: 1 \leq s, t \leq m_{n}, 1 \leq i, j \leq k_{n}, q \in Q_{n+1}, p\right.$ the minimal projection in $Z \cap A_{n}$ with $\left.q=q p\right\}$.

Then $A_{n+1}$ is an abelian subalgebra of $M$ with minimal projections elements of its spanning set, $A_{n} \subseteq A_{n+1}$, and $A_{n+1} \subseteq N_{n+1}$. Further $N_{n+1}$ is a finite dimensional subalgebra of $M$ which is a direct sum over $q \in Q_{n+1}$ of algebras each of which is isomorphic to $\mathrm{M}_{k_{n+1}}(\mathrm{C})$ with matrix units $e_{i 1}^{p} e_{s t}^{q} e_{1 j}^{p}$, where $1 \leq s, t \leq m_{n}, 1 \leq i, j \leq k_{n}$ and $p$ is the unique minimal projection in $Z \cap A_{n}$ such that $q=q p$. This shows that the centre of $N_{n+1}$ is the linear span 
of $Q_{n+1}$ so equals $N_{n+1} \cap Z$. Let $1 \leq s \leq n-1$ and let $r$ be a minimal projection in $Z \cap A_{s}$. Let $u$ be the natural unitary in $N_{s}$ that interchanges $e_{11}^{r}$ and $e_{i i}^{r}$ in $N_{s}$. Then $u$ normalises $A_{n}$ by (6), and so normalises the minimal projections $e_{j 1}^{p} q_{t t}^{q} e_{1 j}^{p}$ spanning $A_{n+1}$. If $u$ is the natural unitary in $N_{n}$ that interchanges $e_{11}^{p}$ and $e_{i i}^{p}$, then $u$ normalises $A_{n+1}$ by definition of the minimal projections in $A_{n+1}$. We have checked conditions (1) to (6) of the induction, and only condition (7) remains to be proved from (8).

Firstly observe that for all $x \in M$,

$$
\left\|\left(E_{A_{n+1}^{\prime} \cap M}-E_{A_{n+1}}\right)\left(e_{j j}^{p} x e_{j j}^{p}\right)\right\|_{2}=\left\|\left(E_{A_{n+1}^{\prime} \cap M}-E_{A_{n+1}}\right)\left(e_{1 j}^{p} x e_{j 1}^{p}\right)\right\|_{2}
$$

because the map $e_{j j}^{p} x e_{j j}^{p} \rightarrow e_{1 j}^{p} x e_{j 1}^{p}$ is an isometric isomorphism from $e_{j j}^{p} M e_{j j}^{p}$ onto $e_{1 j}^{p} M e_{j 1}^{p}$ that carries $e_{j j}^{p} A_{n+1}^{\prime} \cap M e_{j j}^{p}$ onto $e_{1 j}^{p} A_{n+1}^{\prime} \cap M e_{j 1}^{p}$ and $e_{j j}^{p} A_{n+1} e_{j j}^{p}$ onto $e_{1 j}^{p} A_{n+1} e_{1 j}^{p}$.

Now for each $1 \leq i \leq n+1$,

$$
\text { 9. }\left\|\left(E_{A_{n+1}^{\prime} \cap M}-E_{A_{n+1}}\right)\left(x_{i}\right)\right\|_{2}^{2}=\Sigma\left\|e_{j j}^{p}\left(E_{A_{n+1}^{\prime} \cap M}-E_{A_{n+1}}\right)\left(x_{i}\right) e_{j j}^{p}\right\|_{2}^{2} \text {, }
$$

where the summation is over $1 \leq j \leq k_{n}$ and all minimal projections $p$ in $A_{n} \cap Z$, and equality holds because of the orthorgonality of $e_{j j}^{p} M e_{j j}^{p}$, for different $j$ and $p$, and $e_{j j}^{p}$ being in the commutants of the two algebras $A_{n+1}^{\prime} \cap M$ and $A_{n+1}$. Further $e_{j j}^{p}$ is in these two algebras so (9) equals

$$
\Sigma\left\|\left.\left(E_{A_{n+1}^{\prime} \cap M}-E_{A_{n+1}}\right)\left(e_{j j}^{p} x_{i} e_{j j}^{p}\right)\right|_{2} ^{2}=\Sigma\right\|\left(E_{A_{n+1}^{\prime} \cap M}-E_{\left.A_{n+1}\right)}\left(e_{1 j}^{p} p x_{i} e_{j 1}^{p}\right) \|_{2}^{2}\right.
$$

by the note above on the isometries. Now $e_{11}^{p} A_{n+1}^{\prime} \cap M e_{11}^{p}=e_{11}^{p} B_{\ell}^{\prime} \cap M e_{11}^{p}$ and $e_{11}^{p} A_{n+1} e_{11}^{p}=e_{11}^{p} B_{\ell} e_{11}^{p}$ by the definition of $A_{n+1}$ in terms of $B_{\ell}$ and its minimal projections. Thus (9) equals

$$
\Sigma||\left(E_{B_{\ell}^{\prime} \cap M}-E_{B_{\ell}}\right)\left(e_{1 j}^{p} x_{i} e_{j 1}^{p}\right) \|_{2}^{2} \leq \Sigma 4^{-(n+1)} \delta_{n}^{-1} k_{n}^{-1}=4^{-(n+1)}
$$

as the summation extends over $\delta_{n}$ minimal projections $p$ in $Z \cap A_{n}$ and $k_{n}$ subscripts since $\left\|e_{1 j}^{p} y e_{j 1}^{p}\right\|_{2}=\left\|e_{j j}^{p} y e_{j j}^{p}\right\|_{2}$ for all $p$ and $j$. This finishes the inductive construction.

Let $N=\left(\cup N_{n}\right)^{-w}$ and $A=\left(\cup A_{n}\right)^{-w}$, where ${ }^{-w}$ denotes weak closure. Clearly $A$ is self-adjoint abelian, $N$ is injective and $A \subseteq N$. By (7) and the $\|\cdot\|_{2}$-density of $\left\{x_{i}: i \geq 0\right\}$ in the closed unit ball $M_{1}$, it follows that $\left\|\left(E_{A_{n}^{\prime} \cap M}-E_{A_{n}}\right)(x)\right\|_{2}$ tends to zero for all $x \in M$. Hence $A$ is a masa by [Po 1, Lemma 1.2]. Now $N^{\prime} \cap M \subseteq A^{\prime} \cap M=A$, since $A$ is a masa in $M$, so $N^{\prime} \cap M \subseteq N^{\prime} \cap A \subseteq N^{\prime} \cap N$, which equals $Z$ by (2) and Lemma 7. This proves Theorem 8 . 


\section{Injectives containing Cartan subalgebras}

THeOREM 9. Let $M$ be a type $I_{1}$ von Neumann algebra with separable predual and let $A$ be a Cartan subalgebra of $M$. Then there is an injective von Neumann subalgebra $N$ of $M$ with relative commutant the centre $Z$ of $M$ such that $A$ is a Cartan subalgebra of $N$.

The hypothesis that $A$ is a Cartan subalgebra of a type $I I_{1}$ von Neumann algebra $M$ applies in the following discussion and definitions and in Lemma 10.

Let $\mathscr{N}(A)$ be the normalizer of $A$ in the unitary group of $M$, so $u \in \mathscr{N}(A)$ if and only if $u A u^{*}=A$. Let

$$
\mathscr{G}=\{v \in M: v=u p, u \in \mathscr{N}(A), p \in P(A)\},
$$

so elements of $\mathscr{G}$ are partial isometrics with $v^{*} v=p \in A$ and $v v^{*}=u p u^{*} \in A$. Observe that $\mathscr{G}$ is a semigroup in $M$ as $v=u p$ and $t=w q$ implies that $v t=u p w q=u w \cdot w^{*} p w \cdot q$ with $u w \in \mathscr{N}(A)$ and $w^{*} p w \cdot q$ a projection in $A$ since $A$ is abelian and $w^{*} p w \in A$. Further $\mathscr{G}^{*}=\mathscr{G}$ as $v=u p$ implies $v^{*}=u^{*} \cdot u p u^{*}$.

Lemma 10. Let $f_{1}, \ldots, f_{k}$ be a set of orthogonal equivalent projections in $A$. Then there are matrix units $f_{i j}$ in $\mathscr{G}$ such that $f_{i i}=f_{i}$ for $1 \leq i \leq k$.

Proof. The first stage is to show that if $e_{1}$ and $e_{2}$ are equivalent orthogonal projections in $\mathrm{A}$ then there is a $v \in \mathscr{G}$ with $v^{*} v=e_{1}$ and $v v^{*}=e_{2}$. The construction of $v$ is done by a standard maximal trick; this part of the proof is exactly as in the first part of the proof of [Po 1, Prop 3.6], which is repeated here for the readers convenience.

Let $\mathscr{F}=\left\{(p, w): p \in P(A), w \in \mathscr{G}, p \leq e_{1}, w^{*} w=p\right.$ and $\left.w w^{*} \leq e_{2}\right\}$. Note $\mathscr{F}$ is not empty since $(0,0) \in \mathscr{F}$. Define a partial order $\leq$ on $\mathscr{F}$ by $\left(p_{1}, w_{1}\right) \leq\left(p_{2}, w_{2}\right)$ if and only if $p_{1} \leq p_{2}$ and $w_{1}=w_{2} p_{1}$. By Zorn's Lemma we obtain a maximal totally ordered subfamily $\mathscr{F}_{o}$ of $\mathscr{F}$ (which has a countable cofinal subfamily since $M$ has separable predual), and let $(p, v) \in \mathscr{F}$ be the maximal element of $\mathscr{F}_{o}$. Suppose $p \neq e_{1}$. Let $p_{1}=e_{1}-p=e_{1}-v^{*} v$ and $q_{1}=e_{2}-v v^{*}$. Note that $p_{1}$ and $q_{1}$ are equivalent projections - this can be seen easily here using the central trace $T$ as $T\left(p_{1}\right)=T\left(q_{1}\right)$.

If $p_{1} u q_{1} u^{*}=0$ for each unitary $u \in \mathscr{N}(A)$, then the projection $g=\vee\left\{u q_{1} u^{*}: u \in \mathscr{N}(A)\right\}$ satisfies $p_{1} g=0, q_{1} g=q_{1}$ and $g \in P(Z)$, because $\mathscr{N}(A)$ generates $M$ as a von Neumann algebra. Now $T\left(p_{1}\right)=T\left(q_{1}\right)=$ $T\left(q_{1}\right) g=T\left(p_{1}\right) g=T\left(p_{1} g\right)=0$ contrary to $p_{1} \neq 0$, where the first and third equalities hold because $p_{1} \sim q_{1}$. This contradiction implies that $p_{1} u q_{1} u^{*}=p_{o} \neq 0$ for some $u \in \mathscr{N}(A)$. Now $p_{o} \leq p_{1}$ and $u p_{1} \in \mathscr{G}$ by construction of $\mathscr{G}$. If $v=w p$ with $w \in \mathscr{N}(A)($ recall $(p, v) \in \mathscr{F})$, then $v+u p_{o} \in \mathscr{G}$ 
because $p+p_{o} \in P(A)\left(p p_{o}=0\right)$ and there is a unitary $u_{1} \in \mathcal{N}(A)$ such that $u_{1}\left(p+p_{o}\right)=v+u p_{o}$. Hence $\left(p+p_{o}, v+u p_{o}\right) \in \mathscr{F}$ and is strictly greater than $(p, v)$, contradicting the maximality of the element $(p, v)$. Hence $v \in \mathscr{G}$ satisfies $v^{*} v=e_{1}(=p)$ and $v v^{*}=e_{2}$.

Using the above choose $f_{1 j}(1 \leq j \leq k)$ in $\mathscr{G}$ such that $f_{1 j} f_{1 j} *=f_{1}$ and $f_{1 j}^{*} f_{1 j}=f_{j}$ for $1 \leq j \leq k$. Define $f_{i j}=f_{1 j}^{*} f_{1 j}$ for $1 \leq i, j \leq k$. These $f_{i j}$ are the required matrix units in $\mathscr{G}$.

Proof of Theorem 9. Let $\left\{a_{n}: n \in \mathrm{N}\right\}$ be a sequence of projections in $A$ whose linear span is dense in $A$ in the strong topology. Observe that $A$ has such a sequence, because $M$ and hence $A$ has separable predual. Further note that as $\tau$ is a faithful normal tracial state on $M$, density in the $\|\cdot\|_{2}$ topology implies strong density in the unit ball of $A$.

By induction we construct an increasing sequence $N_{o}=\mathrm{C} 1 \subset \mathrm{C} N_{1} \subset$ $\mathrm{CN}_{2} \ldots$ of finite dimensional *-subalgebras of $M$ with matrix units $e_{i j}^{q}$ in each of the matrix direct summands satisfying

1. the centre of $N_{n}$ is equal to $N_{n} \cap Z$,

2. if $Q(n)$ denotes the set of minimal idempotents in $N_{n} \cap Z$, then the matrix units $e_{i j}^{q}(1 \leq i, j \leq k(q))$ constructed in $q N_{n}$ are in $\mathscr{G}$ for all $q \in Q(n)$,

3. if $A_{n}$ is the finite dimensional subalgebra generated by the set $\left\{e_{i i}^{q}: 1 \leq i \leq k(q), q \in Q(n)\right\}$, then $A_{n} \subset A$, and

4. $\left\|a_{j}-E_{A_{n}}\left(a_{j}\right)\right\|_{2} \leq 2^{-n}$ for $1 \leq j \leq n$.

Recall that $E_{A_{n}}$ denotes the $\tau$ preserving conditional expectation from $M$ onto $A_{n}$. Suppose that $N_{n}$ has been constructed. If $v=u e$ is in $\mathscr{G}$ with $u \in \mathscr{N}(A)$ and $e$ a projection in $\mathrm{A}$, then $v x v^{*}=u e x e u^{*}=u e x u^{*}$ is in $\mathrm{A}$ for all $x \in A$. Thus the finite set

$$
F=\left\{\Sigma_{q} e_{1 i}^{q} a_{t} e_{i 1}^{q}: 1 \leq t \leq n+1,1 \leq i \leq k(q)\right\}
$$

is contained in $\Sigma_{q} e_{11}^{q} A e_{11}^{q}$, where all sums over $q$ run over $Q(n)$. Now by Lemma 5 there is a finite dimensional subalgebra $A_{o}$ of $A$ and $k \in \mathrm{N}$ such that $A_{n} \subseteq A_{o}$,

5. $\left\|\left(I-E_{A_{o}}\right)\left(e_{1 i}^{q} a_{t} e_{i 1}^{q}\right)\right\|_{2} \leq \tau(q)^{\frac{1}{2}} k_{n}^{-1} 2^{-(n+1)}$ for all $q \in Q(n), 1 \leq i \leq k_{n}$ and $1 \leq t \leq n+1$, and

6. if $Z_{n+1}=A_{o} \cap Z$, then for each minimal projection $p$ in $Z_{n+1}$, the minimal projections $f_{1}^{p}, \ldots, f_{k}^{p}$ in $p A_{o}$ are all equivalent.

Let $Q(n+1)$ be the set of minimal projections in $Z_{n+1}$. For each $p \in Q(n+1)$ and each $q \in Q(n)$ with $p q=p$, the projection $p e_{11}^{q}$ is a sum of a finite number of $f_{i}^{p}$, which are the minimal projections in $p A_{o}$. By renumbering the projections $f_{i}^{p}$ (if necessary) we will assume that $p e_{11}^{q}=\Sigma_{1}^{k_{0}(p)} f_{i}^{p}$ for some $k_{o}(p)$ and all $p q=p$. As the projections 
$f_{i}^{p}(1 \leq i \leq k)$ in A are orthogonal and equivalent, there is a set of matrix units $f_{i j}^{p}$ in $\mathscr{G}$ so that $f_{i i}^{p}=f_{i}^{p}$ for $1 \leq i \leq k$ by Lemma 10 . The matrix units $e_{\ell m}^{p}$ are defined by a tensor operation using $f_{i j}^{p}, e_{s 1}^{q}, e_{1 t}^{q}$ for $p q=p$ and all $i, j, s, t$ :

7. Let $e_{i, j, s, t}^{p}=e_{s 1}^{q} f_{i j}^{p} q_{1 t}^{q}$ for $p=q p(p \in Q(n+1), q \in Q(n)), 1 \leq i, j \leq k_{o}(p)$ and $1 \leq s, t \leq k(q)$.

Let $N_{n+1}$ be the linear span of all $e_{i, j, s, t}^{p}$ defined in (7). Note that $N_{n+1}$ is a *-subalgebra of $M$ and that for each $p \in Q(n+1), e_{i, j, s, t}^{p}$ are the matrix units of $p N_{n+1}$ corresponding to the tensor product $\mathrm{M}_{k_{o}(p)}(\mathrm{C}) \otimes \mathrm{M}_{k(q)}(\mathrm{C})$ with $1 \leq i, j \leq k_{o}(p)$ and $1 \leq s, t \leq k(q)$. Clearly the centre of $N_{n+1}$ is the linear span of $Q(n+1)$ so is $Z_{n+1}$. By construction $e_{i, j, s, t}^{q}$ is in $\mathscr{G}$, because $\mathscr{G}$ is a semigroup under multiplication. The matrix element $e_{i, i, s, s}^{p}$ is of the form uefe $u^{*}$ with $u \in \mathscr{N}(A)$ and $e, f \in P(A)$, because $e_{i i}^{q} \in P(A)$ and $e_{1 s}^{q}=\left(e_{s, 1}^{q}\right)^{*}$, so is in $A$. Let $A_{n+1}$ be the linear span of $e_{i, i, s, s}^{p}$ over all $1 \leq i \leq k_{n}, 1 \leq s \leq k$, $p=p q, p \in Q(n+1)$ and $q \in Q(n)$. Let $k(p)=k_{o}(p) k(q)$. Lastly we check inequality (4) as (1), (2) and (3) have been done for $n+1$. Observe that $e_{11}^{q} A_{o} e_{11}^{q} \subset A_{n+1}$ for all $q \in Q(n)$ as the minimal projections in $e_{11}^{q} A_{o} e_{11}^{q}$ are the projections $e_{i, i, 1,1}^{p}$ for $1 \leq i \leq k_{o}(p)$ and $p q=p$ with $p \in Q(n+1)$. Now note that for each $q \in Q(n)$,

8. $\left\|\left(I-E_{A_{n+1}}\right)\left(e_{i i}^{q} x e_{i i}^{q}\right)\right\|_{2}=\left\|\left(I-E_{A_{o}}\right)\left(e_{1 i}^{q} x e_{i 1}^{q}\right)\right\|_{2}$;

because dropping the $q$ 's, the map $L_{e_{i 1}} R_{e_{1 i}}(L=$ left multiplication, $R=$ right multiplication) from $e_{1 i} A e_{i 1}$ onto $e_{i i} A e_{i i}$ is an isometry since

$$
\left\|L_{e_{i 1}} R_{e_{1 i}}\left(e_{1 i} x e_{i 1}\right)\right\|_{2}^{2}=\tau\left(e_{i 1} e_{1 i} x e_{i 1} e_{1 i} e_{i 1} e_{1 i} x^{*} e_{i 1} e_{1 i}\right)=\left\|e_{i i} x e_{i i}\right\|_{2}^{2} .
$$

Now $e_{i i}^{q} e_{j j}^{r}=0$ if $q \neq r \in Q(n)$, or $q=r$ and $i \neq j$. The module property of the conditional expectation map $E_{A_{n+1}}$ with respect to $e_{i i}^{q} \in A_{n+1}$ implies that the spaces $\left(I-E_{A_{n+1}}\right)\left(e_{i i}^{q} A e_{i i}^{q}\right)$ are orthogonal in $\|\cdot\|_{2}$ norm for different pairs $(q, i)$. Hence

$$
\left\|\left(I-E_{A_{n+1}}\right)\left(a_{t}\right)\right\|_{2}^{2}=\Sigma\left\|\left(I-E_{A_{n+1}}\right)\left(e_{i i}^{q} a_{t} e_{i i}^{q}\right)\right\|_{2}^{2},
$$

with the sum running over all $q \in Q(n)$ and all $1 \leq i \leq k_{n}$. Equation (8) implies that

$$
\begin{aligned}
& \left\|\left(I-E_{A_{n+1}}\right)\left(a_{t}\right)\right\|_{2}^{2} \\
& =\Sigma\left\|\left(I-E_{A_{o}}\right)\left(e_{1 i}^{q} a_{t} e_{i 1}^{q}\right)\right\|_{2}^{2} \\
& \leq \Sigma \tau(q) k_{n}^{-1} \cdot 4^{-(n+1)} \\
& =4^{-(n+1)} \\
& \text { for } 1 \leq t \leq n+1 \text { by (5) }
\end{aligned}
$$

on recalling that $\Sigma\{q: q \in Q(n)\}=1$ and that $1 \leq i \leq k_{n}$.

The inductive construction of $Z_{n+1}, A_{n+1}, N_{n+1}$ is done with properties (1)(4). Let $N=\left(\cup N_{n}\right)^{-w}$ and $B=\left(\cup A_{n}\right)^{-w}$, where ${ }^{-w}$ denotes the weak closure. 
Then $B \subseteq N, B \subseteq A$, and by (4), $B=A$ since the $\|\cdot\|_{2}$ density of the unit ball of $B$ in that of $A$ implies the weak density.

Now $N^{\prime} \cap M \subset A^{\prime} \cap M=A$, since $\mathrm{A}$ is maximal abelian in $M$; thus $N^{\prime} \cap M=N^{\prime} \cap A=N^{\prime} \cap N$. By (1) of the inductive construction, the sequence $N_{n}$ satisfies the hypotheses of Lemma 7 so that $N^{\prime} \cap N=Z$ and $N$ has trivial relative commutant in $M$ as required.

\section{REFERENCES}

[Ch] E. Christensen, Subalgebras of a finite algebra, Math. Ann. 243 (1979), 17-29.

[CPSS] E. Christensen, F. Pop, A.M. Sinclair and R.R. Smith, On the cohomology groups of certain finite von Neumann algebras, Math. Ann. 306 (1996), 71-92.

[CES] E. Christensen, E.G. Effros and A.M. Sinclair, Completely bounded maps and $C^{*}$-algebraic cohomology, Invent. Math. 90 (1987), 279-296.

[CS1] E. Christensen and A.M. Sinclair, A survey of completely bounded operators, Bull. London Math. Soc. 21 (1989), 417-448.

[CS2] E. Christensen and A.M. Sinclair, On the Hochschild cohomology for von Neumann algebras, preprint (1989).

[Co] A. Connes, Classification of injective factors, Ann. of Math. 104 (1976), 73-116.

[Di] J. Dixmier, les algèbres d'opérateurs dans l'espace Hilbertien, Gauthier-Villars, Paris, 1969.

[Ha] U. Haagerup, The Grothendieck inequality for bilinear forms on $C^{*}$-algebras, Adv. in Math. 56 (1985) 93-116.

[KR] R.V. Kadison and J.R. Ringrose, Fundamentals of the theory of operator algebras, Academic Press, New York, 1983.

[Pi,1] G. Pisier, Grothendieck's Theorem for non-commutative $C^{*}$-algebras with an appendix on Grothendieck's constants, J. Funct. Anal. 29 (1978), 397-415.

[Pi,2] G. Pisier, Factorization of linear operators and geometry of Banach spaces, CBMS Series No. 60, Amer. Math. Soc. Publ., 1986.

[PS] F. Pop and R.R. Smith, Cohomology for certain finite factors, Bull. London Math. Soc. 26 (1994), 303-308.

[Po,1] S. Popa, On a problem of R.V. Kadison on maximal abelian *-subalgebras in factors, Invent. Math. 65 (1981), 269-289.

[Po,2] S. Popa, Notes on Cartan subalgebras in type II factors, Math. Scand. 57 (1985), 171188.

[Ri] J.R. Ringrose, Cohomology of operator algebras, in Lectures on operator algebras, 355434, Lecture Notes in Math. 247, 1972.

[St] S. Stratila, Modular theory, Abacus Press, Tunbridge Wells, England 1981.

[SZ] S. Stratila and L. Zsido, Lectures in von Neumann algebras, Abacus Press, Tunbridge Wells, England 1979.

[Ta,1] M. Takesaki, Conditional expectation in von Neumann algebra, J. Funct. Anal. 9 (1972), 306-321.

[Ta,2] M. Takesaki, Theory of operator algebras I, Springer-Verlag, New York 1979.

DEPARTMENT OF MATHEMATICS AND STATISTICS UNIVERSITY OF EDINBURGH EDINBURGH, EH9 3JZ.

U.K.
DEPARTMENT OF MATHEMATICS

TEXAS A\&M UNIVERSITY

COLLEGE STATION, TX 77843

USA 